ESAIM: Proceedings, Vol. 4, 1998, 153-170

Contrôle et Équations aux Dérivées Partielles

http://www.emath.fr/proc/Vol.4/

\title{
LOCAL EXACT CONTROLLABILITY FOR THE 2-D BOUSSINESQ EQUATIONS WITH THE NAVIER SLIP BOUNDARY CONDITIONS.
}

\author{
OLEG YU.IMANUVILOV ${ }^{1}$ \\ Korea Institute for Advanced Study \\ 207-43 Chungryangri-dong Dongdaemoon-ku \\ Seoul Korea 130-012 \\ E-mail: oleg@kias.kaist.ac.kr
}

Key Words : Boussinesq Equation, Local Exact Controllability.

AMS Subject Qualification : 76D05, 49J20, 93B05, 93C20.

\footnotetext{
${ }^{1}$ This research was supported by grant KIAS- M97003.
}

Article published by EDP Sciences and available at http://www.edpsciences.org/proc or http://dx.doi.org/10.1051/proc:1998026 


\begin{abstract}
For distributed controls we get a local exact controllability for the 2-D Boussinesq equations in the case where the fluid is incompressible and slips on the boundary in agreement with the Navier slip boundary conditions.
\end{abstract}




\section{Introduction}

This paper is devoted to a proof of the local exact controllability of the 2-D Boussinesq system with Navier slip boundary conditions, defined in a bounded domain $\Omega \subset R^{2}$ when the control function is distributed on an arbitrary fixed subdomain $\omega \subset \Omega$. The precise statement of the investigated problems and formulations on the main results are placed in section 1 . Here we restrict ourselves only by description of one typical particular case.

Let $(\hat{v}(x), \hat{\theta}(x), \nabla \hat{p}(x)), \quad x \in \Omega \subset R^{2}$ be a steady-state solution of the 2-D Boussinesq system:

$$
\begin{gathered}
-\Delta \hat{v}+(\hat{v}, \nabla) \hat{v}+\vec{e} \hat{\theta}+\nabla \hat{p}=f(x), \operatorname{div} \hat{v}=0,\left.(\hat{v}, \nu)\right|_{\partial \Omega}=0, \operatorname{rot} \hat{v}+\sigma(\hat{v}, \tau)=0, \\
-\Delta \hat{\theta}+(\hat{v}, \nabla \hat{\theta})+(\hat{v}, \vec{e})=h(x),\left.\quad \hat{\theta}\right|_{\partial \Omega}=0 .
\end{gathered}
$$

We consider the nonstationary Boussinesq system

$$
\begin{gathered}
\partial_{t} v(t, x)-\Delta v(t, x)+(v, \nabla) v+\vec{e} \theta+\nabla p=f(x)+u^{\prime}(t, x), \quad \operatorname{div} v=0, \\
\partial_{t} \theta-\Delta \theta+(v, \nabla \theta)+(v, \vec{e})=h(t, x)+u_{3}(t, x),
\end{gathered}
$$

with boundary conditions

$$
\left.(v, \nu)\right|_{\partial \Omega}=0,\left.\quad(\operatorname{rot} v+\sigma(v, \tau))\right|_{\partial \Omega}=0,\left.\quad \theta\right|_{\partial \Omega}=0
$$

and initial conditions

$$
\left.v\right|_{t=0}=v_{0}(x),\left.\quad \theta\right|_{t=0}=\theta_{0}(x),
$$

which is sufficiently closed to a given steady-state solution

$$
\left\|v_{0}-\hat{v}\right\|_{\left(W_{2}^{2}(\Omega)\right)^{2}}+\left\|\theta_{0}-\hat{\theta}\right\|_{W_{2}^{1}(\Omega)} \leq \varepsilon, \quad \text { ( parameter } \varepsilon \text { is sufficiently small). }
$$

One has to find a locally distributed control

$$
\operatorname{supp} u \subset \omega,
$$

such that the solution $(v(t, x), \theta(t, x))$ of boundary value problem (3)- $(7)$ at the prescribed instant $T$ coincides with $(\hat{v}, \hat{\theta}):(v(T, x), \theta(T, x)) \equiv(\hat{v}(x), \hat{\theta}(x))$. Such control is constructed in this work.

To make this result more clear let us assume, that $(\hat{v}, \hat{\theta})$ satisfies (1), (2) and $(\hat{v}, \hat{\theta})$ is an unstable singular point of the dynamical system generated by equation (3), (4) in the phase space of solenoidal vector fields with the Navier slip conditions on $\partial \Omega$. Let $\left(v_{0}, \theta_{0}\right)$ be an initial condition from the neighborhood of $(\hat{v}, \hat{\theta})$ such that the trajectory of the dynamical system going out $\left(v_{0}, \theta_{0}\right)$ does not converge to $\left(v_{0}, \theta_{0}\right)$ as $t \rightarrow \infty$. As we show in this work one can construct boundary control, such that the corresponding trajectory going out $\left(v_{0}, \theta_{0}\right)$ reaches $\hat{v}, \hat{\theta}$ during a finite time. In other words, one can suppress a 
turbulence rise by means of the boundary control. This result makes more clear the question on connections between turbulence and controllability [17].

The global approximate controllability of the Navier-Stokes equations with the slip boundary conditions was obtained by J.-M. Coron [3]. The proof is based on ideas which were succesfully applied in [2], [4] to solve exact controllability problem for the Euler equation. Unfortunately, expect the case $\bar{\sigma}=0$ approximate controllability is proved in the sence of weak norm. So here we can not combine this result with the local exact controllability one as it was made in [5]. Works of A.V.Fursikov, O.Yu. Imanuvilov [7]-[12], A.V. Fursikov [6] precedes to this paper. In [8] the local exact controllability for the Burgers equation was studied. The case of the 2-D and 3-D Navier-Stokes system with control on the hole boundary and $\hat{v}=0$ was investigated in [7] and [6] respectively. Papers [11], [12] are concerned on local exact controllability of the Boussinesq system. The controllability of 2-D Navier-Stokes equations with slip boundary conditions for the case $\sigma(x) \equiv 0$ was studied in [9].

\section{Statement of the problem and formulation of the main results.}

1.1. In a bounded simply connected domain $\Omega \subset R^{2}$ with boundary $\partial \Omega \in C^{\infty}$ we consider the Boussinesq system

$$
\begin{gathered}
\partial_{t} v(t, x)-\Delta v(t, x)+(v, \nabla) v+\vec{e} \theta+\nabla p(t, x)=f(t, x)+u^{\prime}(t, x), \\
\partial_{t} \theta-\Delta \theta+(v, \nabla \theta)+(v, \vec{e})=h(t, x)+u_{3}(t, x), \\
\operatorname{div} v=\partial_{x_{1}} v_{1}+\partial_{x_{2}} v_{2}=0, \quad \operatorname{supp} u \subset \omega,
\end{gathered}
$$

where $(t, x) \in Q \equiv(0, T) \times \Omega, v(t, x)=\left(v_{1}(t, x), v_{2}(t, x)\right)$ is a velocity of the fluid, $\theta(t, x)$ is a temperature of the fluid, $\nabla p(t, x)$ is a pressure gradient, $\partial_{t}=$ $\frac{\partial}{\partial t}, \partial_{x_{j}}=\frac{\partial}{\partial x_{j}},(v, \nabla) v=\sum_{j=1}^{2} v_{j} \partial_{x_{j}} v, \quad \Delta$ is the Laplace operator, $f=\left(f_{1}, f_{2}\right)$ is a density of external forces, $h(t, x)$ is a density of external heat sources, $\vec{e}$ is the vector of gravity force direction, $\omega \subset \Omega$ is an arbitrary fixed subdomain and $u(t, x)=\left(u^{\prime}, u_{3}\right)=\left(u_{1}(t, x), u_{2}(t, x), u_{3}(t, x)\right)$ is a control function. We assume that

$$
\left.v(t, x)\right|_{t=0}=v_{0}(x),\left.\quad \theta(t, x)\right|_{t=0}=\theta_{0}(x),
$$

where $v_{0}(x)=\left(v_{01}(x), v_{02}(x)\right), \theta_{0}(x)$ is a given initial conditions.

We set on $\Sigma=(0, T) \times \partial \Omega$ the Navier slip boundary conditions

$$
\left.(\operatorname{rot} v+\sigma(v, \tau))\right|_{\Sigma}=0,\left.\quad(v, \nu)\right|_{\Sigma}=0,
$$

and the Dirichlet zero boundary condition for temperature

$$
\left.\theta\right|_{\partial \Omega}=0
$$


where $\nu=\left(\nu_{1}, \nu_{2}\right)$ is the vector field of outward unit normals to $\partial \Omega, \tau=\left(\tau_{1}, \tau_{2}\right)$ is the unit tangent vector field on $\partial \Omega, \sigma(x) \in C^{\infty}(\partial \Omega)$ defined by

$$
\sigma(x)=\frac{2(1-\bar{\sigma}) k(x)-\bar{\sigma}}{1-\bar{\sigma}},
$$

where $k$ is the curvature of $\partial \Omega$ defined through the relation $\frac{\partial n}{\partial \tau}=k \tau$. We recall that $(v, \nu)=v_{1} \nu_{1}+v_{2} \nu_{2}, \operatorname{rot} v=\partial_{x_{1}} v_{2}-\partial_{x_{2}} v_{1}$.

To set the problem and formulate the main results we have to introduce the functional spaces. Recall, that $W_{p}^{k}(\Omega), k \geq 0,1 \leq p<\infty$ is the Sobolev space of functions with finite norm

$$
\|u\|_{W_{p}^{k}(\Omega)}=\left(\sum_{|\alpha| \leq k} \int_{\Omega}\left|\partial^{|\alpha|} u(x) / \partial x_{1}^{\alpha_{1}} \partial x_{2}^{\alpha_{2}}\right|^{p} d x\right)^{1 / p},
$$

where $\alpha=\left(\alpha_{1}, \alpha_{2}\right),|\alpha|=\alpha_{1}+\alpha_{2}$.

We set

$$
\begin{gathered}
V^{k}(\Omega)=\left\{v(x)=\left(v_{1}, v_{2}\right) \in\left(W_{2}^{k}(\Omega)\right)^{2}: \operatorname{div} v=0\right\}, \\
W^{1,2(k)}(Q)=\left\{v \in L_{2}\left(0, T ; W_{2}^{k+2}(\Omega)\right): \partial_{t} v \in L_{2}\left(0, T ; W_{2}^{k}(\Omega)\right)\right\}, \\
V^{1,2(k)}(Q)=\left\{v(t, x) \in\left(W^{1,2(k)}(Q)\right): \operatorname{div} v=0\right\} .
\end{gathered}
$$

Since $\nabla p$ can be determined easily from (1.1) by $f, v, \theta$, below keeping in mind solutions of system (1.1)-(1.3) we write $(v, \theta)$ instead of $(v, \theta, \nabla p)$.

Now we set the exact controllability problem. Let a solution $(\hat{v}, \hat{\theta}) \in V^{1,2(1)}(Q) \times$ $W^{1,2(0)}(Q)$ of equation (1.1), (1.2), (1.5), (1.6) as well as an initial condition $\left(v_{0}, \theta_{0}\right) \in V^{2}(\Omega) \times W_{2}^{1}(\Omega)$ be given. We suppose that $\hat{v}, v_{0}, \hat{\theta}, \theta_{0}$ satisfy the inequality

$$
\left\|\hat{v}(0, \cdot)-v_{0}\right\|_{V^{2}(\Omega)}^{2}+\left\|\hat{\theta}(0, \cdot)-\theta_{0}\right\|_{W_{2}^{1}(\Omega)}^{2}<\varepsilon,
$$

where $\varepsilon>0$ is sufficiently small. Assume also that the initial datum $\left(v_{0}, \theta_{0}\right)$ satisfies the compatibility conditions

$$
\left.\left(\operatorname{rot} v_{0}+\sigma\left(v_{0}, \tau\right)\right)\right|_{\partial \Omega}=0,\left.\quad\left(v_{0}, \nu\right)\right|_{\partial \Omega}=0,\left.\quad \theta_{0}\right|_{\partial \Omega}=0 .
$$

The local exact controllability problem is to find a control $u \in\left(L^{2}(Q)\right)^{3}$, such that the solution $(v, \theta) \in V^{1,2(1)}(Q) \times W^{1,2(0)}(Q)$ of (1.1)-(1.6) satisfies at $t=T$ the equation

$$
\left.v(t, x)\right|_{t=T}=\left.\hat{v}(T, x) \quad \theta(t, x)\right|_{t=T}=\hat{\theta}(T, x) .
$$

For $\omega \subset \Omega$ we set $Q^{\omega}=(0, T) \times \omega$.

Theorem 1.1 Let $\partial \Omega$ be connected, $(\hat{v}(t, x), \hat{\theta}(t, x)) \in V^{1,2(1)}(Q) \times W^{1,2(0)}(Q)$ be a given solution of (1.1), (1.2), (1.4)-(1.6) and $\left(v_{0}(x), \theta_{0}(x)\right) \in V^{2}(\Omega) \times$ $W_{2}^{1}(\Omega)$ satisfies (1.10) (1.11) with sufficiently small $\varepsilon>0$. Then there exists a local distributed control $u(t, x) \in\left(L_{2}(Q)\right)^{3}$, supp $u \subset Q^{\omega}$, such that the solution $(v(t, x), \theta(t, x)) \in V^{1,2(1)}(Q) \times W^{1,2(0)}(Q)$ of problem (1.1)- (1.6) exists and satisfies (1.12).

ESAIM: Proc., Vol. 4, 1998, 153-170 


\section{Reduction to a linear controllability problem}

2.1. To get rid of pressure we transform the Navier-Stokes system to the equation for the stream function $\psi$ which is connected with velocity field $v(t, x)=$ $\left(v_{1}, v_{2}\right)$ by equations

$$
\partial_{x_{1}} \psi=-v_{2}, \quad \partial_{x_{2}} \psi=v_{1} .
$$

Application the operator $\partial_{x_{2}}$ to the first of equations (1.1) and $\partial_{x_{1}}$ to the second one, adding this two new equations yields the equation for the stream function:

$\partial_{t}(-\Delta \psi(t, x))+\Delta^{2} \psi+\partial_{x_{2}}\left(\left(\partial_{x_{1}} \psi\right) \Delta \psi\right)-\partial_{x_{1}}\left(\left(\partial_{x_{2}} \psi\right) \Delta \psi\right)+e_{2} \partial_{x_{1}} \theta-e_{1} \partial_{x_{2}} \theta=u_{1}+g$.

We substituted $u_{1}(t, x)+g(t, x)$, instead of rot $f$ in the right-hand-side of $(2.2)$ taking into account that $g=\operatorname{rot} f$ and $u_{1}$ is a control. Just this form of righthand-side we use below. Using the stream function one can rewrite the equation (1.2) as follows

$$
\partial_{t} \theta-\Delta \theta+\partial_{x_{2}} \psi \partial_{x_{1}} \theta-\partial_{x_{1}} \psi \partial_{x_{2}} \theta+\partial_{x_{2}} \psi e_{1}-\partial_{x_{1}} \psi e_{2}=h(t, x)+u_{2}(t, x) .
$$

The first boundary condition from (1.5) by virtue of (2.1) can be rewritten as follows:

$$
\left.\left(-\Delta \psi+\sigma \frac{\partial \psi}{\partial \nu}\right)\right|_{\Sigma}=0, \quad \Sigma=(0, T) \times \partial \Omega .
$$

The second one is transformed to the equation

$$
\left.\partial_{\tau} \psi\right|_{\Sigma}=0
$$

where $\tau=\left(\tau_{1}, \tau_{2}\right)=\left(-\nu_{2}, \nu_{1}\right)$ is the vector tangential to the $\partial \Omega$. By this equality

$$
\left.\psi\right|_{\partial \Omega}=\text { const, }
$$

and since $\partial \Omega$ is a connected set, ${ }^{1}$ function $\psi$ can be determined by (2.1) up to constant arbitrariness. We can assume that

$$
\left.\theta\right|_{\Sigma}=\left.\psi\right|_{\Sigma}=0
$$

without the loosing of generality. By virtue of (2.1), (1.5) instead of initial condition (1.4) we have

$$
\left.\psi(t, x)\right|_{t=0}=\psi_{0}(x),\left.\quad \theta(t, x)\right|_{t=0}=\theta_{0}(x),
$$

where $\psi_{0}$ can be determined by the equalities

$$
\partial_{x_{1}} \psi_{0}=-v_{02}, \quad \partial_{x_{2}} \psi_{0}=v_{01} .
$$

According to (1.11), (2.5) the following compatibility conditions should be fulfilled:

$$
\left.\psi_{0}\right|_{\partial \Omega}=0,\left.\quad\left(-\Delta \psi_{0}+\sigma \frac{\partial \psi_{0}}{\partial \nu}\right)\right|_{\partial \Omega}=\left.0 \quad \theta_{0}\right|_{\partial \Omega}=0 .
$$

\footnotetext{
${ }^{1}$ Only here,deducing condition (2.6) we use connectedness of $\partial \Omega$. Therefore below controllability problem for stream function is studied without assumption of $\partial \Omega$ connectedness. 
Let us assume similarly to section 1 that a solution $(\hat{\psi}(t, x), \hat{\theta}(t, x)) \in W^{1,2(2)}(Q) \times$ $W^{1,2(0)}(Q)$ of equation (2.2), (2.3) with $u(t, x) \equiv 0$ and right side $(g, h) \in$ $\left(L_{2}(Q)\right)^{2}$ is given. Moreover, the function $(\hat{\psi}(t, x), \hat{\theta}(t, x))$ satisfies boundary conditions $(2.4),(2.6)$ and the inequality

$$
\left\|\hat{\psi}(0, \cdot)-\psi_{0}(\cdot)\right\|_{W_{2}^{3}(\Omega)}^{2}+\left\|\hat{\theta}(0, \cdot)-\theta_{0}(\cdot)\right\|_{W_{2}^{1}(\Omega)}^{2}<\varepsilon
$$

holds where $\varepsilon>0$ is sufficiently small.

The local exact controllability problem consists in constructing of such control $u(t, x) \in\left(L_{2}(Q)\right)^{2}, \operatorname{supp} u \subset Q^{\omega}$, that the solution $(\psi(t, x), \theta(t, x))$ of boundary value problem $(2.2),(2.3),(2.4),(2.6),(2.7)$ satisfies the condition

$$
\left.\psi(t, x)\right|_{t=T}=\left.\hat{\psi}(t, x)\right|_{t=T},\left.\quad \theta(t, x)\right|_{t=T}=\left.\hat{\theta}(t, x)\right|_{t=T} .
$$

We are looking for the solution $(\psi(t, x), \theta(t, x))$ in the following form

$$
\psi(t, x)=w(t, x)+\hat{\psi}(t, x), \quad \theta(t, x)=m(t, x)+\hat{\theta}(t, x),
$$

where $w, m$ are new unknown functions. Substitution of (2.11) into (2.2) - (2.7) yields the equation for the function $w$ and $m$ :

$$
\partial_{t}(-\Delta w(t, x))+\Delta^{2} w+B(\hat{\psi}+w, w)+B(w, \hat{\psi})+e_{2} \partial_{x_{1}} \theta-e_{1} \partial_{x_{2}} \theta=u_{1}(t, x),
$$

where

$$
\begin{gathered}
B(\psi, \varphi)=\partial_{x_{2}}\left(\left(\partial_{x_{1}} \psi\right) \Delta \varphi\right)-\partial_{x_{1}}\left(\left(\partial_{x_{2}} \psi\right) \Delta \varphi\right) \\
\partial_{t} m-\Delta m+(\hat{v}, \nabla m)+\left(\nabla^{\perp} w, \nabla m+\vec{e}\right)+\partial_{x_{2}} w \partial_{x_{1}} \hat{\theta}-\partial_{x_{1}} w \partial_{x_{2}} \hat{\theta}=u_{2} .
\end{gathered}
$$

(Here we set $\nabla^{\perp} w=\left(\partial_{x_{2}} w,-\partial_{x_{1}} w\right)$.) This also gives boundary and initial conditions

$$
\begin{gathered}
\left.\left(-\Delta w+\sigma \frac{\partial w}{\partial \nu}\right)\right|_{\Sigma}=0,\left.\quad m\right|_{\Sigma}=\left.w\right|_{\Sigma}=0 \\
\left.w(t, x)\right|_{t=0}=w_{0},\left.\quad m(t, x)\right|_{t=0}=m_{0} .
\end{gathered}
$$

Here $w_{0}(x)=\psi_{0}(x)-\hat{\psi}(0, x), m_{0}(x)=\theta_{0}(x)-\hat{\theta}(0, x)$. By virtue of $(2.11)$, (2.7), (2.8), (2.9) we have

$\left.m_{0}\right|_{\partial \Omega}=\left.w_{0}\right|_{\partial \Omega}=\left.\left(-\Delta w_{0}+\sigma \frac{\partial w_{0}}{\partial \nu}\right)\right|_{\partial \Omega}=0, \quad\left\|w_{0}\right\|_{W_{2}^{3}(\Omega)}^{2}+\left\|m_{0}\right\|_{W_{2}^{1}(\Omega)}^{2}<\varepsilon$.

In Sections 2-4 the following assertion will be proved:

Theorem 2.1. Suppose that $\hat{\psi} \in W^{1,2(2)}(Q), \hat{\theta} \in W^{1,2(0)}(Q)$ satisfies (2.2), (2.3) with $u \equiv 0$, (2.4), (2.6), and initial condition $\left(w_{0}, m_{0}\right) \in W_{2}^{3}(\Omega) \times W_{2}^{1}(\Omega)$ satisfies (2.17) with sufficiently small $\varepsilon>0$. Then one can find such control $u \in\left(L_{2}(Q)\right)^{2}$, supp $u \subset(0, T) \times \omega$, that the corresponding solution $(w, m) \in$ $W^{1,2(2)}(Q) \times W^{1,2(0)}(Q)$ of problem (2.12) -(2.16) exists and satisfies equality

$$
\left.w(t, x)\right|_{t=T}=\left.0 \quad m(t, x)\right|_{t=T}=0 .
$$

ESAIM: Proc., Vol. 4, 1998, 153-170 
2.2. To prove Theorem 2.1 we use the following right inverse operator theorem:

Theorem 2.2. Let $X, Z$ be Banach spaces and

$$
A: X \rightarrow Z
$$

be a continuously differentiated mapping. Assume that for some $x_{0} \in X$, and $z_{0} \in Z$ equality

$$
A\left(x_{0}\right)=z_{0}
$$

holds, and the derivative

$$
A^{\prime}\left(x_{0}\right): X \rightarrow Z
$$

of $A$ at $x_{0}$ is an surjective operator. Then for a sufficiently small $\varepsilon>0$ there exists a mapping $M(z): B_{\varepsilon}\left(z_{0}\right) \rightarrow X$ defined on the ball

$$
B_{\varepsilon}\left(z_{0}\right)=\left\{z \in Z:\left\|z-z_{0}\right\|_{Z}<\varepsilon\right\}
$$

which satisfies conditions

$$
\begin{gathered}
A(M(z))=z, \quad z \in B_{\varepsilon}\left(z_{0}\right), \\
\left\|M(z)-x_{0}\right\|_{X} \leq k\left\|A\left(x_{0}\right)-z\right\|_{Z} \text { for all } \quad z \in B_{\varepsilon}\left(z_{0}\right),
\end{gathered}
$$

where $k>0$ is a certain constant.

The Theorem 2.2 is a simple corollary of generalization of the Implicit function theorem proved in [1].

In our case the space $X$ consists of pairs $(w, m, u)$, and operator $A(x)$ is defined by formulas (2.12), (2.14):

$$
\begin{gathered}
A(x)=\left(-\partial_{t} \Delta w+\Delta^{2} w+B(\hat{\psi}+w, w)+B(w, \hat{\psi})-u_{1},\right. \\
\partial_{t} m-\Delta m+\left(\hat{v}+\nabla^{\perp} w, \nabla m\right)+\partial_{x_{2}} \psi \partial_{x_{1}} \hat{\theta}-\partial_{x_{1}} \psi \partial_{x_{2}} \hat{\theta}+\partial_{x_{2}} w e_{1}-\partial_{x_{1}} w e_{2}-u_{2}, \\
\left.\left.w\right|_{t=0},\left.m\right|_{t=0}\right)
\end{gathered}
$$

( the condition $\left.w\right|_{t=T}=0,\left.m\right|_{t=T}=0$ and boundary conditions for $w, m$ will be included to the space $X$ definition. ) The space $Z$ will be determined by set of pairs from (2.24). Set $x_{0}=(0,0), z_{0}=(0,0)$. Evidently, equality $(2.20)$ is fulfilled.

To check the epimorphism condition of operator (2.21) we write out the equation

$$
A^{\prime}\left(x_{0}\right) x=z .
$$

In our case this equation is as follows:

$$
\begin{array}{r}
L(w, m)-u \equiv \partial_{t}(-\Delta w)+\Delta^{2} w+B(\hat{\psi}, w)+B(w, \hat{\psi})+e_{2} \partial_{x_{1}} m-e_{1} \partial_{x_{2}} m-u_{1}=f \\
N(w, m)-u \equiv \partial_{t} m-\Delta m+(\hat{v}, \nabla m)+\left(\nabla^{\perp} w, \nabla \hat{\theta}+\vec{e}\right)-u_{2}=h,
\end{array}
$$


where $u=\chi_{\omega} u, \chi_{\omega}$ is the characteristic function of the set $\omega\left(\chi_{\omega}(x)=1\right.$ for $x \in$ $\omega ; \chi_{\omega}(x)=0$ for $\left.x \in \Omega \backslash \omega\right)$,

$$
\begin{gathered}
\left.m\right|_{\Sigma}=\left.w\right|_{\Sigma}=\left.\left(-\Delta w+\sigma \frac{\partial w}{\partial \nu}\right)\right|_{\Sigma}=0, \\
\left.m\right|_{t=0}=m_{0},\left.\quad w\right|_{t=0}=w_{0},\left.\quad m\right|_{t=T}=\left.w\right|_{t=T}=0 .
\end{gathered}
$$

We start from the following lemma:

LemmA 2.1 Let $\omega_{0} \subset \subset \omega$ be an arbitrary fixed subdomain of $\Omega$. Then there exists a function $\beta \in C^{2}(\bar{\Omega})$ such that

$$
\beta(x)>0 \forall x \in \Omega,\left.\beta\right|_{\partial \Omega}=0, \quad|\nabla \beta(x)|>0 \quad \forall x \in \Omega \backslash \omega_{0} .
$$

For the proof of this lemma see [15].

Set

$$
\begin{array}{r}
\eta(t, x) \equiv \eta^{\lambda}(t, x)=\left(e^{\lambda^{2}\|\beta\|_{C(\bar{\Omega})}}-e^{\lambda \beta(x)}\right) /((T-t) \ell(t))^{3}, \\
\bar{\eta}(t)=\min _{x \in \Omega} \eta(t, x)=\left(e^{\lambda^{2}\|\beta\|_{C(\bar{\Omega})}}-e^{\lambda \max _{x} \in \Omega}\right) /((T-t) \ell(t))^{3},
\end{array}
$$

where $\lambda>1$ is a parameter (magnitude of $\lambda$ will be fixed below), function $\beta(x)$ defined in Lemma 2.1 and function $\ell(t) \in C^{\infty}[0, T]$ satisfies conditions

$$
\ell(t)>0, \forall t \in[0, T], \ell(t)=t \forall t \in[T / 2, T] .
$$

Let us assume that $\lambda>1$ such that

$$
49 \min _{x \in \Omega} \eta(t, x) \geq 48 \max _{x \in \Omega} \eta(t, x)=\left(e^{\lambda^{2}\|\beta\|_{C(\bar{\Omega})}}-1\right) /((T-t) \ell(t))^{3} .
$$

Obviously inequality (2.31) holds true for all $\lambda$ sufficiently large. Finally we define the parameter $\lambda$ in Lemma 3.1. Set

$$
L_{2}(Q, \rho)=\left\{u(t, x),(t, x) \in Q:\|u\|_{L_{2}(Q, \rho)}^{2} \equiv \int_{Q} \rho^{2}(t, x) u^{2}(t, x) d x d t<\infty\right\} .
$$

The weight functions $\rho$ used below are constructed by means of functions (2.30). One of such weight functions is defined by the formula

$$
\kappa(t, x)=\left\{\begin{array}{cr}
e^{s \eta} & x \in \Omega \backslash \omega \\
1 & x \in \omega,
\end{array}\right.
$$

where parameter $s>0$ will be defined in Lemma 4.3. We introduce the space

$$
\begin{gathered}
Y(Q) \equiv\left\{(y(t, x), z(t, x)) \in\left(W^{1,2(2)}(Q) \times W^{1,2(0)}(Q)\right):\right. \\
\left.y\right|_{\Sigma}=\left.z\right|_{\Sigma}=\left.\left(-\Delta y+\sigma \frac{\partial y}{\partial \nu}\right)\right|_{\Sigma}=0,
\end{gathered}
$$

ESAIM: Proc., Vol. 4, 1998, 153-170 


$$
\begin{gathered}
\|(y, z)\|_{Y(Q)}^{2} \equiv\left\|\partial_{t}(-\Delta y)+\Delta^{2} y+B(\hat{\psi}, y)+B(y, \hat{\psi})+e_{2} \partial_{x_{1}} z-e_{1} \partial_{x_{2}} z\right\|_{L_{2}(Q, \kappa)}^{2} \\
+\left\|\partial_{t} z-\Delta z+(\hat{v}, \nabla z)+\partial_{x_{2}} y \partial_{x_{1}} \hat{\theta}-\partial_{x_{1}} y \partial_{x_{2}} \hat{\theta}+\partial_{x_{2}} y e_{1}-\partial_{x_{1}} y e_{2}\right\|_{L_{2}(Q, \kappa)}^{2} \\
\left.+\left\|e^{\frac{98}{100} s \bar{\eta}} y\right\|_{W^{1,2(2)}(Q)}^{2}+\left\|e^{\frac{98}{100} s \bar{\eta}} z\right\|_{W^{1,2(0)}(Q)}^{2}<\infty\right\}
\end{gathered}
$$

where functions $\kappa, \eta$ are defined in (2.33), (2.30) and parameter $s$ from (2.33). Define also

$$
U_{\omega}(Q)=\left\{u(t, x) \in\left(L_{2}(Q)\right)^{2}: \operatorname{supp} u \subset Q^{\omega}\right\}
$$

where, remind, $Q^{\omega}=(0, T) \times \omega$.

To apply the Theorem 2.2 in order to establish solvability of (2.12), (2.14), (2.15), (2.16), (2.18) we define spaces $X, Z$ as follows:

$$
\begin{gathered}
X=\left\{(y, z, u) \in Y(Q) \times U_{\omega}(Q):\right. \\
\left.\|(y, z, u)\|_{X}^{2}=\|(y, z)\|_{Y(Q)}^{2}+\|u\|_{U_{\omega}(Q)}^{2}<\infty\right\}, \\
Z=\left(L_{2}(Q, \kappa)\right)^{2} \times \hat{W}_{2}^{3}(\Omega) \times \stackrel{\circ}{W_{2}^{1}(\Omega),}
\end{gathered}
$$

where

$$
\begin{aligned}
& \hat{W}_{2}^{3}(\Omega)=\left\{v(x) \in W_{2}^{3}(\Omega):\left.v\right|_{\partial \Omega}=\left.\left(-\Delta v+\sigma \frac{\partial v}{\partial \nu}\right)\right|_{\partial \Omega}=0\right\} . \\
& \stackrel{\circ}{W_{2}^{1}}(\Omega)=\left\{v(x) \in W_{2}^{1}(\Omega),\left.v\right|_{\partial \Omega}=0\right\} .
\end{aligned}
$$

We have

Proposition 2.1 Let the spaces $X, Y, Z$ be defined in (2.34), (2.36), (2.37) operator $A(x)$ be defined by (2.24). Then mapping (2.19) is continuously differentiated for any point $x_{0} \in X$.

Evidently, equality (2.20) holds if $A$ is mapping (2.24), $x_{0}=\left(w^{0}, u^{0}\right)=$ $0, z_{0}=0$. So, to apply Theorem 2.2 we have to establish only that image of operator (2.21) coincides with $Z$. This is reduced to the proof of problem (2.25)-(2.28) solvability for any $\left(f, h, w_{0}, \theta_{0}\right) \in Z$. Sections $3-4$ are devoted to achievement of this aim. 


\section{Carleman estimate for the heat equation.}

This section is devoted to solve observability problem for the operator $\left(L^{*}, N^{*}\right)$. We start from Carleman estimate for the inverse heat equation. Of course Carleman estimates for such equation is well know in the case of function with compact support [16] or for the heat equation with zero Dirichlet or Neumann boundary conditions [13]-[15]. But here we do not introduce boundary conditions on $\Sigma$. We set

$$
\begin{gathered}
\varphi(t, x)=e^{\lambda \beta(x)} /(t(T-t))^{3}, \\
\alpha(t, x)=\left(e^{\lambda \beta}-e^{\lambda^{2}\|\beta\|_{C(\bar{\Omega})}}\right) /(t(T-t))^{3}
\end{gathered}
$$

where $\lambda>1$ satisfies (2.31) and function $\beta$ from Lemma 2.1.

In the cylinder $Q$ we consider the heat equation:

$$
G z=\partial_{t} z+\Delta z=f(t, x) \text { in } Q,
$$

Let $\omega_{0} \subset \subset \omega_{1} \subset \subset \omega$. We have

LEMmA 3.1 There exists a number $\hat{\lambda}>0$ such that for an arbitrary $\lambda>\hat{\lambda}$ there exists $s_{0}(\lambda)>0$ that for any $s>s_{0}$ the solution $z(t, x)$ of (3.3) satisfies the Carleman estimate:

$$
\begin{gathered}
\int_{Q}\left((s \varphi)^{-1}\left|\frac{\partial z}{\partial t}\right|^{2}+s \varphi \sum_{j=1}^{n}\left|\frac{\partial z}{\partial x_{i}}\right|^{2}+s^{3} \varphi^{3} z^{2}\right) e^{2 s \alpha(t, x)} d x d t \\
\leq c\left(\int_{Q} f^{2}(t, x) e^{2 s \alpha} d x d t\right. \\
\left.+\int_{\Sigma}\left(\left|\frac{\partial z}{\partial t} \frac{\partial z}{\partial \nu}\right|+s^{2} \varphi^{2}|\nabla z||z|\right) e^{2 s \alpha} d \Sigma+\int_{Q^{\omega_{1}}} s^{3} \varphi^{3} z^{2} e^{2 s \alpha} d x d t\right),
\end{gathered}
$$

where the functions $\varphi(t, x), \alpha(t, x)$ are defined in (3.1), (3.2), and $c>0$ does not depend on $s$.

Let us consider the Dirichlet boundary value problem for the Laplace operator

$$
\Delta \psi=z \text { in } \Omega,\left.\quad \psi\right|_{\partial \Omega}=0
$$

We have

LEMmA 3.2 There exists a number $\hat{\lambda}>0$ such that for an arbitrary $\lambda>\hat{\lambda}$ there exists $s_{0}(\lambda)$ such that for each $s \geq s_{0}(\lambda)$ the solutions of problem (3.5) satisfy the following inequality

$$
\begin{gathered}
\int_{\Omega}\left(\frac{1}{s \varphi}\left(\sum_{i, j=1}^{n}\left|\frac{\partial^{2} \psi}{\partial x_{i} \partial x_{j}}\right|^{2}\right)+s \varphi|\nabla \psi|^{2}+s^{3} \varphi^{3} \psi^{2}\right) e^{2 s \alpha} d x+\int_{\Sigma} s \varphi\left(\frac{\partial \psi}{\partial \nu}\right)^{2} e^{2 s \alpha} d \Sigma \\
\leq c_{1}\left(\int_{\Omega}|z|^{2} e^{2 s \alpha} d x+\int_{\omega_{1}} s^{3} \varphi^{3} \psi^{2} e^{2 s \alpha} d x d t\right)
\end{gathered}
$$

The proof of this Lemma see for example in [14], [10].

ESAIM: Proc., Vol. 4, 1998, 153-170 
Now we consider the parabolic equation

$$
\begin{gathered}
L^{*}(p, q) \equiv \partial_{t}(\Delta p(t, x))+\Delta^{2} p+B_{2}^{*}(\psi, p)+B_{1}^{*}(p, \psi)-\partial_{x_{2}}\left(\left(\partial_{x_{1}} \hat{\theta}+e_{1}\right) q\right)+ \\
\partial_{x_{1}}\left(\left(\partial_{x_{2}} \hat{\theta}+e_{2}\right) q\right)=f \\
N^{*}(p, q) \equiv-\partial_{t} q-\Delta q+(\hat{v}, \nabla q)-\partial_{x_{1}}\left(e_{2} p\right)+\partial_{x_{2}}\left(e_{1} p\right)=h \\
\left.q\right|_{\Sigma}=\left.p\right|_{\Sigma}=\left.\left(-\Delta p+\sigma \frac{\partial p}{\partial \nu}\right)\right|_{\Sigma}=0 \\
p(T, \cdot)=p_{0}, \quad q(T, \cdot)=q_{0}
\end{gathered}
$$

where $B_{1}^{*}(\cdot, \psi), B_{2}^{*}(\psi, \cdot)$ are operators adjoint formally to the linear operators $B(\cdot, \psi), B(\psi, \cdot)$ respectively. By definition $(2.13)$ of the operator $B(\psi, \varphi)$ we have

$B_{1}^{*}(h, \psi)=\partial_{x_{1}}\left(\Delta \psi \partial_{x_{2}} h\right)-\partial_{x_{2}}\left(\Delta \psi \partial_{x_{1}} h\right), B_{2}^{*}(\psi, h)=\Delta\left(\partial_{x_{1}} h \partial_{x_{2}} \psi-\partial_{x_{2}} h \partial_{x_{1}} \psi\right)$.

The following Lemma can be easily proved by the standard energy methods.

Lemma 3.3 Let $f, h \in L^{2}(Q), p_{0} \in \hat{W}_{2}^{3}(\Omega)$ and $q_{0} \in \stackrel{\circ}{W_{2}^{1}}(\Omega)$. Then there exists the unique solution of problem (3.7)-(3.10) which satisfies the estimate

$\|(p, q)\|_{W^{1,2(2)}(Q) \times W^{1,2(0)}(Q)} \leq c\left(\|f\|_{L^{2}(Q)}+\|q\|_{L^{2}(Q)}+\left\|p_{0}\right\|_{W_{2}^{3}(\Omega)}+\left\|q_{0}\right\|_{W_{2}^{1}(\Omega)}\right)$.

We have

LEmma3.4 There exists $s>0$ such that the solutions of the problem (3.7)(3.10) satisfy the estimate

$$
\begin{gathered}
\int_{Q}\left(\frac{1}{((T-t) t)^{3}}\left(|\nabla \Delta p|^{2}+|\nabla q|^{2}\right)+\frac{1}{((T-t) t)^{9}}\left(|\Delta p|^{2}+q^{2}\right)+\right. \\
\left.\frac{1}{((T-t) t)^{12}}|\nabla p|^{2}+\frac{1}{((T-t) t)^{18}}|p|^{2}\right) e^{2 s \alpha} d x d t \leq C(s)\left(\int_{Q}\left(f^{2}+h^{2}\right) e^{2 s \alpha} d x d t\right. \\
+\int_{Q^{\omega}}\left(\frac{1}{((T-t) t)^{21}} p^{2}+\frac{1}{((T-t) t)^{21}} q^{2}\right) e^{2 s \alpha} d x d t .
\end{gathered}
$$

Proof. Applying the Carleman estimates (3.4), (3.6) to equation (3.7) for all $s$ sufficiently large we obtain

$$
\begin{gathered}
\int_{Q}\left(\frac{1}{s \varphi}\left(\left|\frac{\partial \Delta p}{\partial t}\right|^{2}+\left|\Delta^{2} p\right|^{2}\right)+s \varphi|\nabla \Delta p|^{2}+s^{3} \varphi^{3}|\Delta p|^{2}+s^{4} \varphi^{4}|\nabla p|^{2}+s^{6} \varphi^{6} p^{2}\right) e^{2 s \alpha} d x d t+ \\
\int_{\Sigma}\left(\left|\frac{\partial p t}{\partial \nu}\right|^{2}+s^{4} \varphi^{4}\left|\frac{\partial p}{\partial \nu}\right|^{2}\right) e^{2 s \alpha} d \Sigma \leq C\left(\int_{Q^{\omega}}\left(s^{3} \varphi^{3}|\Delta p|^{2}+s^{6} \varphi^{6}|p|^{2}\right) e^{2 s \alpha} d x d t\right. \\
\left.+\int_{Q} f_{s}^{2} e^{2 s \alpha} d x d t+\int_{\Sigma}\left(\left|\frac{\partial \Delta p}{\partial t} \frac{\partial \Delta p}{\partial \nu}\right|+s^{2} \varphi^{2}|\nabla \Delta p|\left|\frac{\partial p}{\partial \nu}\right|\right) e^{2 s \alpha} d \Sigma\right)
\end{gathered}
$$


where $f_{s}=\partial_{x_{2}}\left(\left(\partial_{x_{1}} \hat{\theta}+e_{1}\right) q\right)-\partial_{x_{1}}\left(\left(\partial_{x_{2}} \hat{\theta}+e_{2}\right) q\right)$. Note, that by $(3.9)$

$$
\left.\frac{\partial \Delta p}{\partial t}\right|_{\Sigma}=\left.\sigma \frac{\partial^{2} p}{\partial t \partial \nu}\right|_{\Sigma}
$$

Then, applying the Cauchy-Bynyakovskii inequality to the last integral in the right hand side of (3.14) we have

$$
\begin{gathered}
\int_{Q}\left(\frac{1}{s \varphi}\left(\left|\frac{\partial \Delta p}{\partial t}\right|^{2}+|\Delta p|^{2}\right)+s \varphi|\nabla \Delta p|^{2}+s^{3} \varphi^{3}|\Delta p|^{2}+s^{4} \varphi^{4}|\nabla p|^{2}+\right. \\
\left.s^{6} \varphi^{6} p^{2}\right) e^{2 s \alpha} d x d t \\
\leq C\left(\int_{Q^{\omega_{1}}}\left(s^{3} \varphi^{3}|\Delta p|^{2}+s^{6} \varphi^{6}|p|^{2}\right) e^{2 s \alpha} d x d t+\int_{\Sigma}\left(\left|\frac{\partial^{2} p}{\partial t \partial \nu}\right|^{2}+|\nabla \Delta p|^{2}\right) e^{2 s \alpha} d \Sigma+\right. \\
\left.\int_{Q} f_{s}^{2} e^{2 s \alpha} d x d t\right) .
\end{gathered}
$$

Let $x_{0} \in \partial \Omega$. Set $\bar{\alpha}(t)=\alpha\left(x_{0}, t\right), g(t, x)=e^{s \bar{\alpha}(t)} p(t, x), r(t, x)=e^{s \bar{\alpha}(t)} f_{s}(t, x)$. The function $g(t, x)$ satisfies equations

$$
\begin{gathered}
\partial_{t}(\Delta g(t, x))-l_{1} \Delta g+\Delta^{2} g+B_{2}^{*}(\psi, g)+B_{1}^{*}(g, \psi)=r, \\
\left.g\right|_{\Sigma}=\left.\left(-\Delta g+\sigma \frac{\partial g}{\partial \nu}\right)\right|_{\Sigma}=0, \\
g(0, \cdot)=g(T, \cdot)=0,
\end{gathered}
$$

where $l_{1}=s \frac{\partial}{\partial t} \bar{\alpha}$. By Lemma 3.3 the solution of problem (3.16)-(3.18) satisfies the estimate

$$
\|g\|_{W^{1,2(2)}(Q)} \leq c\left(\|r\|_{L^{2}(Q)}+\left\|l_{1} \Delta g\right\|_{L^{2}(Q)}\right) .
$$

From (3.19) by (3.15) and the Sobolev imbedding theorem we have

$$
\|\Delta g\|_{L^{2}\left(0, T: W_{2}^{1}(\partial \Omega)\right)}+\left\|\frac{\partial^{2} g}{\partial \nu \partial t}\right\|_{L^{2}(\Sigma)} \leq c\left(\left\|s \varphi^{\frac{4}{3}} \Delta g\right\|_{L^{2}(Q)}+\|r\|_{L^{2}(Q)}\right) .
$$

The inequalities (3.15) and (3.20) yield:

$$
\begin{gathered}
I=\int_{Q}\left(\frac{1}{s \varphi}\left|\frac{\partial \Delta p}{\partial t}\right|^{2}+s \varphi|\nabla \Delta p|^{2}+s^{3} \varphi^{3}|\Delta p|^{2}+s^{4} \varphi^{4}|\nabla p|^{2}+s^{6} \varphi^{6} p^{2}\right) e^{2 s \alpha} d x d t \\
\leq C\left(\int_{Q^{\omega_{1}}}\left(s^{3} \varphi^{3}|\Delta p|^{2}+s^{6} \varphi^{6}|p|^{2}\right) e^{2 s \alpha} d x d t+s \int_{Q} f_{s}^{2} e^{2 s \alpha} d x d t\right)
\end{gathered}
$$

ESAIM: Proc., Vol. 4, 1998, 153-170 
Let $\rho(x) \in C_{0}^{\infty}(\omega),\left.\rho\right|_{\omega_{1}} \equiv 1$. Note that

$$
\begin{gathered}
\int_{Q^{\omega_{1}}} s^{3} \varphi^{3}|\Delta p|^{2} d x d t \leq C \int_{Q^{\omega}} s^{3} \frac{1}{((T-t) t)^{9}} \rho|\Delta p|^{2} d x d t= \\
C \int_{Q^{\omega}} s^{3} \frac{1}{((T-t) t)^{9}}\left(\rho p \Delta^{2} p+2 p(\nabla(\rho p), \nabla \Delta p)+p \Delta \rho \Delta p\right) d x d t \\
\leq C_{1}(\varepsilon) \int_{Q^{\omega}} \frac{s^{7}}{((T-t) t)^{21}} p^{2} d x d t+\varepsilon \int_{Q^{\omega}}\left(\frac{((T-t) t)^{3}}{s}\left|\Delta^{2} p\right|^{2}+\right. \\
\left.\frac{s}{((T-t) t)^{3}}|\nabla \Delta p|^{2}+\frac{s^{3}}{((T-t) t)^{9}}|\Delta p|^{2}\right) d x d t .
\end{gathered}
$$

From (3.21), (3.22) we obtain

$$
I \leq C\left(\int_{Q^{\omega_{1}}} s^{7} \varphi^{7}|p|^{2} e^{2 s \alpha} d x d t+s \int_{Q} f_{s}^{2} e^{2 s \alpha} d x d t\right) .
$$

Applying to (3.8) the carleman estimate (1.6) proved in [10] for all $s$ sufficiently large we have

$$
\int_{Q}\left(s \varphi|\nabla q|^{2}+s^{3} \varphi^{3} q^{2}\right) e^{2 s \alpha} d x d t \leq C\left(\int_{Q^{\omega_{1}}} s^{3} \varphi^{3} q^{3} d x d t+\int_{Q}\left(h^{2}+|\nabla p|^{2}\right) e^{2 s \alpha} d x d t\right) .
$$

Thus the statement of the our Lemma follows from (3.23), (3.24) and estimate (3.12).

\section{Exact controllability of the linearized Boussi- nesq system}

In this section we will prove an existence theorem for the exact controllability problem (2.25)-(2.28). In the previous section we proved estimate (3.13) which solves observability problem for the operator (3.7), (3.8). The following Lemma converts observability result into controllability one.

Lemma 4.1 Let $\hat{\psi} \in W^{1,2(2)}(Q), \hat{\theta} \in W^{1,2(0)}(Q), f, h \in L_{2}\left(Q, e^{s \eta}\right), w_{0} \in$ $\hat{W}_{2}^{3}(\Omega), m_{0} \in \stackrel{\circ}{W_{2}^{1}}(\Omega)$. Then there exists solution of problem (2.25)-(2.28) $(w, m) \in\left(W_{2}^{1,2(0)}(Q) \cap L^{2}\left(Q, e^{s \eta} /(T-t)^{5}\right)\right)^{2}, u \in\left(L^{2}\left(Q, e^{s \eta}\right)\right)^{2}$, supp $\subset \subset Q^{\omega_{0}}$ which satisfies the estimate

$$
\begin{gathered}
\|(w, m, u)\|_{\left(W_{2}^{1,2(0)}(Q) \cap L^{2}\left(Q, e^{s \eta} /(T-t)^{5}\right)\right)^{2} \times\left(L^{2}\left(Q, e^{2 s \eta}\right)\right)^{2}} \\
\leq c\left(\left\|w_{0}\right\|_{W_{2}^{1}(\Omega)}+\left\|m_{0}\right\|_{W_{2}^{1}(\Omega)}+\|h\|_{L^{2}\left(Q, e^{s \eta}\right)}+\|f\|_{L^{2}\left(Q, e^{s \eta}\right)}\right) .
\end{gathered}
$$

The proof of Lemma 4.1 similar to the proof of Theorem 2.1 from [9]. 
Let us consider the initial boundary value problem for the linearized Boussinesq system with the slip boundary conditions

$$
\begin{gathered}
L(w, m)=f, \quad N(w, m)=h \text { in } Q, \\
\left.m\right|_{\partial \Omega}=\left.w\right|_{\partial \Omega}=\left.\left(-\Delta w+\sigma \frac{\partial w}{\partial \nu}\right)\right|_{\partial \Omega}=0, \\
w(0, \cdot)=w_{0}, \quad m(0, \cdot)=m_{0} .
\end{gathered}
$$

We have

Lemma 4.2 Let $\hat{\psi} \in W^{1,2(2)}(Q), \hat{\theta} \in W^{1,2(0)}(Q), w_{0} \in \hat{W}_{2}^{3}(\Omega), m_{0} \in \stackrel{\circ}{W_{2}^{1}}(\Omega)$. Then for an arbitrary $h, f \in L^{2}(Q)$ there exists the unique solution $(w, m) \in$ $W^{1,2(2)}(Q) \times W^{1,2(0)}(Q)$ of the problem (4.2)- (4.4) which satisfies the estimate

$\|(w, m)\|_{W^{1,2(2)}(Q) \times W^{1,2(0)}(Q)} \leq c\left(\left\|w_{0}\right\|_{W_{2}^{3}(\Omega)}+\left\|m_{0}\right\|_{W_{2}^{1}(\Omega)}+\|f\|_{L^{2}(Q)}+\|h\|_{L^{2}(Q)}\right)$.

This lemma can be easily proved by standard energy method.

The main result of this section is the following lemma.

Lemma 4.3 Let $\hat{\psi} \in W^{1,2(2)}(Q), \hat{\theta} \in W^{1,2(0)}(Q), h, f \in L_{2}(Q, \kappa), w_{0} \in$ $\hat{W}_{2}^{3}(\Omega), m_{0} \in \stackrel{\circ}{W_{2}^{1}}(\Omega)$. Then there exists solution of problem (2.25)-(2.28) $(w, m) \in Y(Q) \cap\left(L^{2}\left(Q, e^{s \eta} /(T-t)^{5}\right)\right)^{2}, u \in U_{\omega}$ which satisfies the estimate

$\|(w, m, u)\|_{Y(Q) \times L^{2}(Q)} \leq c\left(\left\|w_{0}\right\|_{W_{2}^{3}(\Omega)}+\left\|m_{0}\right\|_{W_{2}^{1}(\Omega)}+\|f\|_{L^{2}(Q, \kappa)}+\|h\|_{L^{2}(Q, \kappa)}\right)$.

Proof. Note, that without the loosing of generality we can assume that $\left(w_{0}, m_{0}\right) \equiv 0$. Indeed, let $\left(w_{0}, m_{0}\right) \neq 0$. For this case we are looking for solution of problem (2.25)-(2.28) in the form

$$
w(t, x)=\bar{w}(t, x)+\ell_{2}(t) \tilde{w}(t, x), \quad m(t, x)=\bar{m}(t, x)+\ell_{2}(t) \tilde{m}(t, x),
$$

where $(\tilde{w}(t, x), \tilde{m}(t, x))$ is a solution of the boundary value problem (4.2)-(4.4) with initial datum $w_{0}, m_{0}, h \equiv f \equiv 0$, and $\ell_{2}(t)$ is a function which has the following property:

$$
\ell_{2}(t)=1 \forall t \in[0, T / 4], \quad \ell_{2}(t)=0 \forall t \in[3 T / 4, T] .
$$

The function $(\bar{w}, \bar{m})$ is a solution of exact controllability problem

$$
\begin{gathered}
L(\bar{w}, \bar{m})=L\left(\ell_{2} \tilde{w}, \ell_{2} \tilde{m}\right)+f+u_{1}, \quad N(\bar{w}, \bar{m})=N\left(\ell_{2} \tilde{w}, \ell_{2} \tilde{m}\right)+h+u_{2} \text { in } Q \\
\left.\bar{m}\right|_{\partial \Omega}=\left.\bar{w}\right|_{\partial \Omega}=\left.\left(-\Delta \bar{w}+\sigma \frac{\partial \bar{w}}{\partial \nu}\right)\right|_{\partial \Omega}=0, \\
\bar{w}(0, \cdot)=0, \quad \bar{m}(0, \cdot)=0, \quad \bar{w}(T, \cdot)=0, \quad \bar{m}(T, \cdot)=0 .
\end{gathered}
$$

ESAIM: Proc., Vol. 4, 1998, 153-170 
From Lemma 4.2 we get $\left(L\left(\ell_{2} \tilde{w}, \ell_{2} \tilde{m}\right), N\left(\ell_{2} \tilde{w}, \ell_{2} \tilde{m}\right)\right) \in\left(L^{2}(Q)\right)^{2}$. By virtue of (4.7) $\left(L\left(\ell_{2} \tilde{w}, \ell_{2} \tilde{m}\right), N\left(\ell_{2} \tilde{w}, \ell_{2} \tilde{m}\right)\right) \in\left(L^{2}(Q, \kappa)\right)^{2}$. Hence we reduced the our original problem to problem (2.25)-(2.28) with initial datum $m_{0} \equiv w_{0} \equiv 0, h, f \in$ $L^{2}(Q, \kappa)$.

Now let us assume that $h, f \in L^{2}\left(Q, e^{s \eta}\right)$. By virtue of Lemma 4.1 there exists solution of the problem (2.25)-(2.28) $(w, m, u)$ which satisfies the estimate (4.1). Denote $q(t, x)=e^{s \bar{\eta}(t)} w(t, x), z(t, x)=e^{s \bar{\eta}(t)} m(t, x)$, where $\bar{\eta}(t)$ is defined in (2.30). Then the pair $(q, z)$ satisfies the system of equations

$$
\begin{gathered}
L(q, z)=-s \bar{\eta}_{t} \Delta q+\left(u_{1}+f\right) e^{s \bar{\eta}}, N(q, z)=s \bar{\eta}_{t} z+\left(u_{2}+h\right) e^{s \bar{\eta}} \text { in } Q, \\
\left.z\right|_{\partial \Omega}=\left.q\right|_{\partial \Omega}=\left.\left(-\Delta q+\sigma \frac{\partial q}{\partial \nu}\right)\right|_{\partial \Omega}=0, \\
q(0, \cdot)=0, z(0, \cdot)=0 .
\end{gathered}
$$

Multiplying equation $(4.8)$ by $(q, z)$ scalarly in $\left(L^{2}(\Omega)\right)^{2}$ and integrating by parts we obtain

$$
\begin{aligned}
& \frac{1}{2} \frac{d}{d t}\left(\|\nabla q\|_{L^{2}(\Omega)}^{2}+\|z\|_{L^{2}(\Omega)}^{2}\right)+\|\Delta q\|_{L^{2}(\Omega)}^{2}+\|\nabla z\|_{L^{2}(\Omega)}^{2} \leq \frac{1}{2}\|\Delta q\|_{L^{2}(\Omega)}^{2}+\frac{1}{2}\|\nabla z\|_{L^{2}(\Omega)}^{2} \\
& +c\left(\|\nabla q\|_{L^{2}(\Omega)}^{2}+\|z\|_{L^{2}(\Omega)}^{2}+\left\|(u+(f, h)) e^{s \bar{\eta}}\right\|_{L^{2}(\Omega)}^{2}+\left\|s \bar{\eta}_{t} q\right\|_{L^{2}(\Omega)}^{2}+\left\|s \bar{\eta}_{t} z\right\|_{L^{2}(\Omega)}^{2}\right) .
\end{aligned}
$$

Then (4.1), (4.11), (4.5) and Gronwall's inequality imply

$$
\|z\|_{L^{2}\left(0, T ; W_{2}^{1}(\Omega)\right) \cap C\left(0, T ; L^{2}(\Omega)\right)}+\|q\|_{C\left(0, T ; W_{2}^{1}(\Omega)\right) \cap L^{2}\left(0, T ; W_{2}^{2}(\Omega)\right)} \leq c .
$$

Denote $\tilde{q}(t, x)=w e^{\frac{99 s \bar{\eta}}{100}}, \tilde{z}(t, x)=z e^{\frac{99 s \bar{\eta}}{100}}, \tilde{f}(t, x)=f e^{\frac{99 s \bar{\eta}}{100}}, \tilde{h}(t, x)=h e^{\frac{99 s \bar{\eta}}{100}}$, $\tilde{u}(t, x)=u e^{\frac{99 s \bar{\eta}}{100}}$. By (4.2)-(4.4) the pair $(\tilde{q}, \tilde{z})$ satisfies the system of equations

$$
\begin{gathered}
L(\tilde{q}, \tilde{z})=-s \frac{99}{100} \bar{\eta}_{t} \Delta \tilde{q}+\tilde{u}_{1}+\tilde{f}, \quad N(\tilde{q}, \tilde{z})=s \frac{99}{100} \bar{\eta}_{t} \tilde{z}+\tilde{u}_{2}+\tilde{h} \text { in } Q, \\
\left.\tilde{z}\right|_{\partial \Omega}=\left.\tilde{q}\right|_{\partial \Omega}=\left.\left(-\Delta \tilde{q}+\sigma \frac{\partial \tilde{q}}{\partial \nu}\right)\right|_{\partial \Omega}=0, \\
\tilde{q}(0, \cdot)=0, \quad \tilde{z}(0, \cdot)=0,
\end{gathered}
$$

where $\tilde{u} \in\left(L^{2}(Q)\right)^{2}, \operatorname{supp} \tilde{u} \subset Q^{\omega}$. Then by virtue of Lemma 4.2

$$
(\tilde{q}, \tilde{z}) \in W^{1,2(2)}(Q) \times W^{1,2(0)}(Q) .
$$

Now let $h(t, x), f(t, x) \in L^{2}(Q, \kappa)$ be an arbitrary functions. We can write it in the form

$$
f(t, x)=\chi_{\omega} f(t, x)+\left(1-\chi_{\omega}\right) f(t, x), h(t, x)=\chi_{\omega} h(t, x)+\left(1-\chi_{\omega}\right) h(t, x) .
$$

By definition of the space $L^{2}(Q, \kappa)$ the functions $\left(1-\chi_{\omega}\right) f,\left(1-\chi_{\omega}\right) h$ belong to $L^{2}\left(Q, e^{s \eta}\right)$. Hence there exists a solution $(w, m, u) \in Y(Q) \times U_{\omega}(Q)$ of problem (2.25)-(2.28) for initial data $\left(w_{0}, m_{0},\left(1-\chi_{\omega}\right) f,\left(1-\chi_{\omega}\right) h\right)$. Obviously 
the pair $\left(w, m, u+\left(\chi_{\omega} f, \chi_{\omega} h\right)\right)$ is the solution of $(2.25)-(2.28)$ for initial date $\left(w_{0}, m_{0}, f, h\right)$.

\section{References}

1. Alekseev V.M., Tikhomirov V.M. and Fomin S.V. Optimal control. Conlsultants Bureau, New York, 1987.

2. Coron J.-M. Contrôlabilité exacte frontiere de l'équation d'Euler des fluids parfaits incompresibles bidimensionnesls.

C.R.Acad. Sci., Paris. T. 317. Série I. 1993, p. 271-276.

3. Coron J.-M. On the Controllability of the 2-D incompressible NavierStokes Equations with the Navier-Slip boundary conditions. ESIAM: Control, Optimization and Calculus of Variations, 1, 1996, p. 35-75.

4. Coron J.-M. On the controllability of 2-D incompressible perfect fluids. J. Math. Pures et Appl. 75, 1996, p. 155-188.

5. Coron J.-M. and Fursikov A.V. Global exact controllability of the 2D Navier-Stokes equations on manifold without boundary. Russian Journal Of Math. Physics, 4, \# 3, 1996, p. 1-20.

6. Fursikov A.V. Exact boundary zero controllability of three dimensional Navier-Stokes equations. J. of Dynamical and Control Syst., 1, \# 3, 1995, p. 325-350.

7. Fursikov A.V. and Imanuvilov O.Yu. On exact boundary zero-controllability of two-dimensional Navier-Stokes equations. Acta Applicandae Mathematicae, 37, 1994, p. 67-76.

8. Fursikov A.V. and Imanuvilov O.Yu. On controllability of certain systems simulating a fluid flow. in Flow Control, IMA vol. Math. Appl., 68, 1995, Ed. by M. D. Gunzburger, Springer-Verberg, New York, p. 148-184.

9. Fursikov A.V. and Imanuvilov O.Yu. Local exact controllability for 2-D Navier-Stokes equations. Sbornik:Mathematics 187, \# 9, 1996, p. 1355-1390.

10. Fursikov A.V. and Imanuvilov O.Yu. Controllability of evolution equations. Lecture Notes Series 34, Recearch Institute of Mathematics Global Analysis Research Center, 1996.

11. Fursikov A.V. and Imanuvilov O.Yu. Local exact controllability of the Navier-Stokes equations. C.R. Acad. Sci. Paris, 323, Série I, 1996, p. 275-280.

12. Fursikov A.V. and Imanuvilov O.Yu. Local exact controllability of the Boussinesq equation. SIAM J. Cont. Optim., (to appear)

13. Imanuvilov O.Yu. Exact boundary controllability of the parabolic equation. Russian Math. Surveys 48, 1993, p. 211-212.

14. Imanuvilov O.Yu. Exact controllability of the semilinear parabolic equation. Vestnik R.U.D.N. ser. Math. 1, 1994, p. 109-116.(in Russian)

15. Imanuvilov O.Yu. Boundary controllability of parabolic equations. Russian Acad. Sci. Sb. Math., 186, \# 6, 1995, p. 109-132. 
16. Isakov V. Carleman type estimates in an anisotropic case and applications. J. Diff. Equ. 8, 1992, p. 193-206.

17. Lions J.-L. Are there connections between turbulence and controllability? $9^{e}$ Conférence internationale de l'INRIA. Antibes. 12-15 juin 1990 